\title{
A Rare Case of Corynebacterium propinquum Endocarditis in a Native Valve
}

\author{
Kevin Kuriakose ${ }^{\mathrm{a}, \mathrm{c}}$, Joshua Rutland ${ }^{\mathrm{a}}$, Thomas E. Watts ${ }^{\mathrm{a}}$, Kshitij Chatterjee ${ }^{\mathrm{a}}$, \\ Yogita Rochlani ${ }^{\mathrm{a}}$, Matthew Burns ${ }^{\mathrm{b}}$
}

\begin{abstract}
We report a case of a 63 -year-old male who presented with complaints of 9 days of fevers up to $38.3{ }^{\circ} \mathrm{C}$, chills and rigors, and headache of 3 4 days duration. He also noticed that the entire right half of his visual field was obscured. MRI of the brain revealed a $4 \mathrm{~cm}$ acute infarction involving the calcarine cortex of the left occipital lobe. Blood cultures grew Corynebacterium propinquum. A trans-esophageal echocardiogram revealed oscillating echodensities on the aortic valve, mitral valve, and posterior leaflet of the tricuspid valve, concerning for infective endocarditis. Repeat bubble study was consistent with a small patent foramen ovale. To the best of our knowledge, there have only been four reported cases of infection by this organism, with just two of them being infective endocarditis. There are no established guidelines for therapy. We used an Etest with minimum inhibitory concentrations (MICs) reported to guide therapy. The patient was successfully treated with 6 weeks of intravenous ceftriaxone.
\end{abstract}

Keywords: Corynebacterium propinquum; Endocarditis; Echocardiogram

\section{Introduction}

Corynebacterium species are a rare cause of infective endocarditis, even more so in native valves. We present a case of native valve infective endocarditis caused by Corynebacterium propinquum that presented with an acute visual field defect. To the best of our knowledge, only four cases of infection by this organism have been reported since the first one in 1994, with just two of them being infective endocarditis. We hope that this case contributes to what little we know of this entity, and per-

Manuscript accepted for publication March 17, 2017

${ }^{a}$ Department of Medicine, University of Arkansas for Medical Sciences, Little Rock, AR, USA

${ }^{b}$ Division of Infectious Diseases, Department of Medicine, University of Arkansas for Medical Sciences, Little Rock, AR, USA

${ }^{\mathrm{c}}$ Corresponding Author: Kevin Kuriakose, 1161 21st Avenue South, \#A-2200, Nashville, TN 37232, USA. Email: kkuriakose6@gmail.com

doi: https://doi.org/10.14740/jmc2795w haps help clinicians make treatment decisions if encountered by a similar presentation.

\section{Case Report}

A 63-year-old Caucasian male with a history of type 2 diabetes mellitus, obstructive sleep apnea, and chronic obstructive pulmonary disease was admitted in early December with complaints of 9 days of fevers up to $38.3{ }^{\circ} \mathrm{C}$, chills and rigors, and headache of 3 - 4 days duration. He initially felt like he had a viral infection. He woke up one day noticing that the entire right half of his visual field was obscured. This prompted him to come to the emergency department. He reported no neck stiffness or body aches, respiratory, or urinary symptoms. His headache was dull and diffuse. He endorsed several episodes of watery, non-bloody stools over the last few days prior to presentation. He also reported contact with a neighbor who had a persistent cough for the past month.

On presentation, he was found to be afebrile, with a normal white cell count of $9,400 / \mathrm{mL}$. His basic metabolic panel (BMP) was only remarkable for a sodium of $132 \mathrm{mEq} / \mathrm{L}$. Computed tomography (CT) of the head without contrast was unremarkable. Neurology service examined the patient in the emergency department and assessed that his vision loss was consistent with a right homonymous hemianopsia. The remainder of his physical exam including cardiovascular and skin exam was completely normal.

After admission, a magnetic resonance angiogram (MRA) of the neck was performed which was unremarkable with normal carotid arteries. MRI of the brain revealed a $4 \mathrm{~cm}$ acute infarction involving the calcarine cortex of the left occipital lobe. Blood cultures on admission were negative, showing no growth at that time and patient had no further episodes of diarrhea or fever since admission. His white cell count remained in the normal range. A transthoracic echocardiogram was done on hospital day 4 as part of workup for embolic stroke. This showed moderate left ventricular systolic dysfunction with an estimated ejection fraction of $35-40 \%$, moderate hypokinesis of the inferior and inferoseptal wall, dilated inferior vena cava with normal respirophasic variation, grossly thickened and calcified aortic valve with mild aortic stenosis, dilated aortic root $(4.2 \mathrm{~cm})$, mild aortic regurgitation, normal mitral and tricuspid valves, and a small very late right to left shunt (14th cardiac cycle) on bubble study consistent with a possible pulmonary 


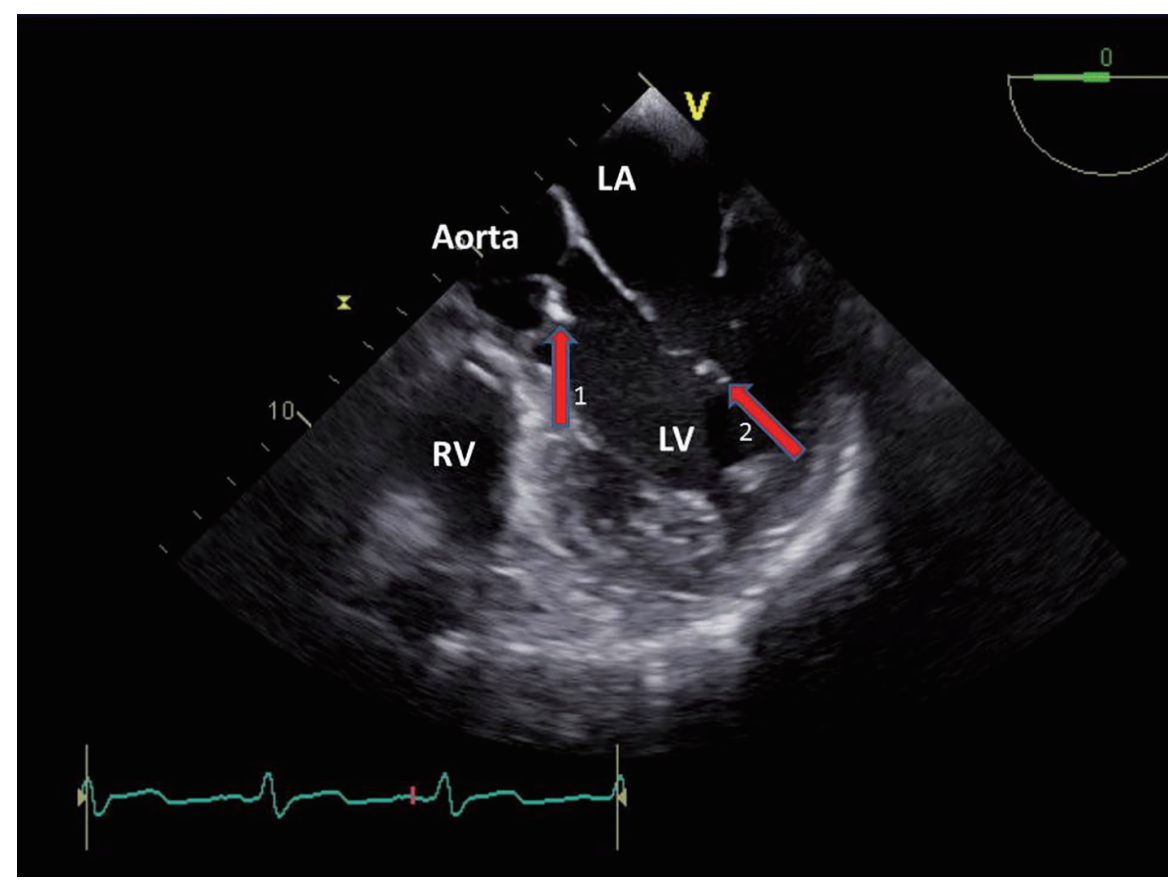

Figure 1. Trans-esophageal echocardiogram revealed echodensities on the aortic (1) and mitral (2) valves.

arterio-venous malformation. His blood cultures then turned positive from two separate sites, growing gram positive bacilli. The organism was eventually identified as C. propinquum.

A trans-esophageal echocardiogram performed the following day (hospital day 5) showed a bicuspid aortic valve with oscillating echodensities on the aortic surface of the valve

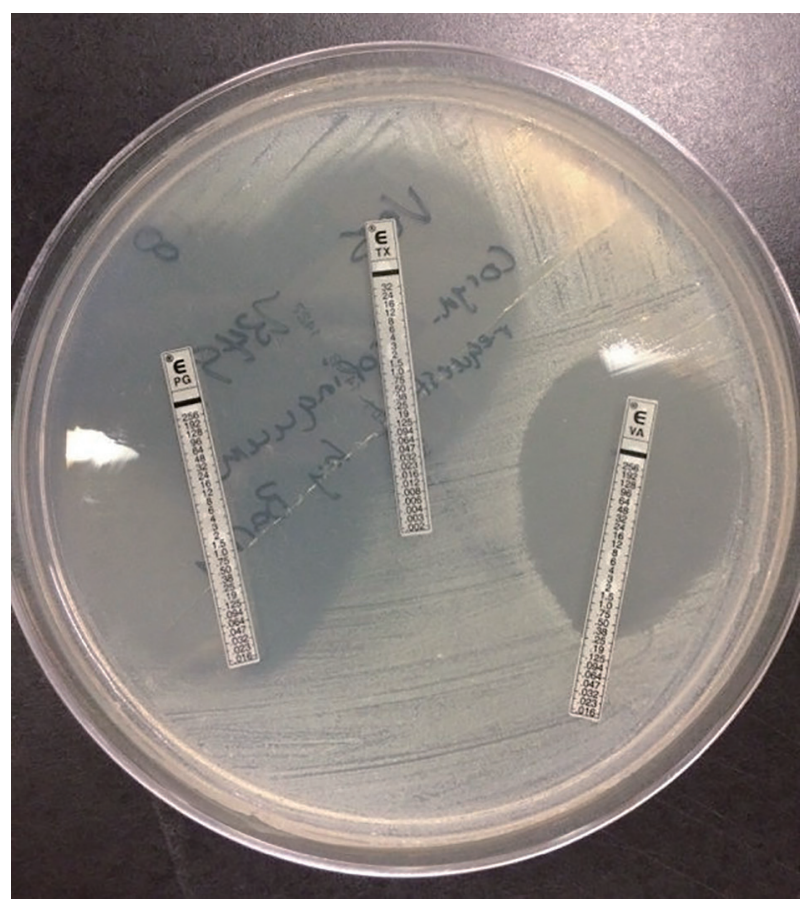

Figure 2. Etest with zones of clearance around penicillin, ceftriaxone, and vancomycin.
$(3 \times 3 \mathrm{~mm})$, anterior leaflet of the mitral valve $(4 \times 4 \mathrm{~mm})$, and posterior leaflet of the tricuspid valve (Fig. 1). Repeat bubble study was consistent with a small patent foramen ovale as bubbles were seen in the left atrium on the third cardiac cycle. There was no obvious atrial septal defect noted.

Infectious diseases, cardiothoracic surgery, and cardiology services were consulted. There was no indication for valve surgery. The patient was initially treated with 3 days of intravenous vancomycin until the bacterium was identified and the susceptibility pattern established. An Etest (bioMerieux) was performed looking at susceptibility to penicillin, ceftriaxone, and vancomycin (Fig. 2). The minimum inhibitory concentrations (MICs) were thought to be acceptable $(<0.016$ for penicillin, 0.032 for ceftriaxone, and 0.38 for vancomycin). Antibiotic therapy was changed to intravenous ceftriaxone monotherapy. The patient was successfully treated with 6 weeks of intravenous ceftriaxone.

He successfully completed antibiotics with resolution of infection. He was doing well with visual adaptation. However, this was complicated by Charles Bonnet syndrome. He was seen in clinic 3 months after initial presentation and noted to be doing well.

\section{Discussion}

This case was noteworthy for two reasons - one being the unusual presentation of infective endocarditis with an acute visual field defect secondary to an embolic infarct of the occipital lobe; and the second being the infective organism - a saprophyte that has previously only been reported to be pathogenic in four other cases.

Infective endocarditis caused by corynebacterial species 


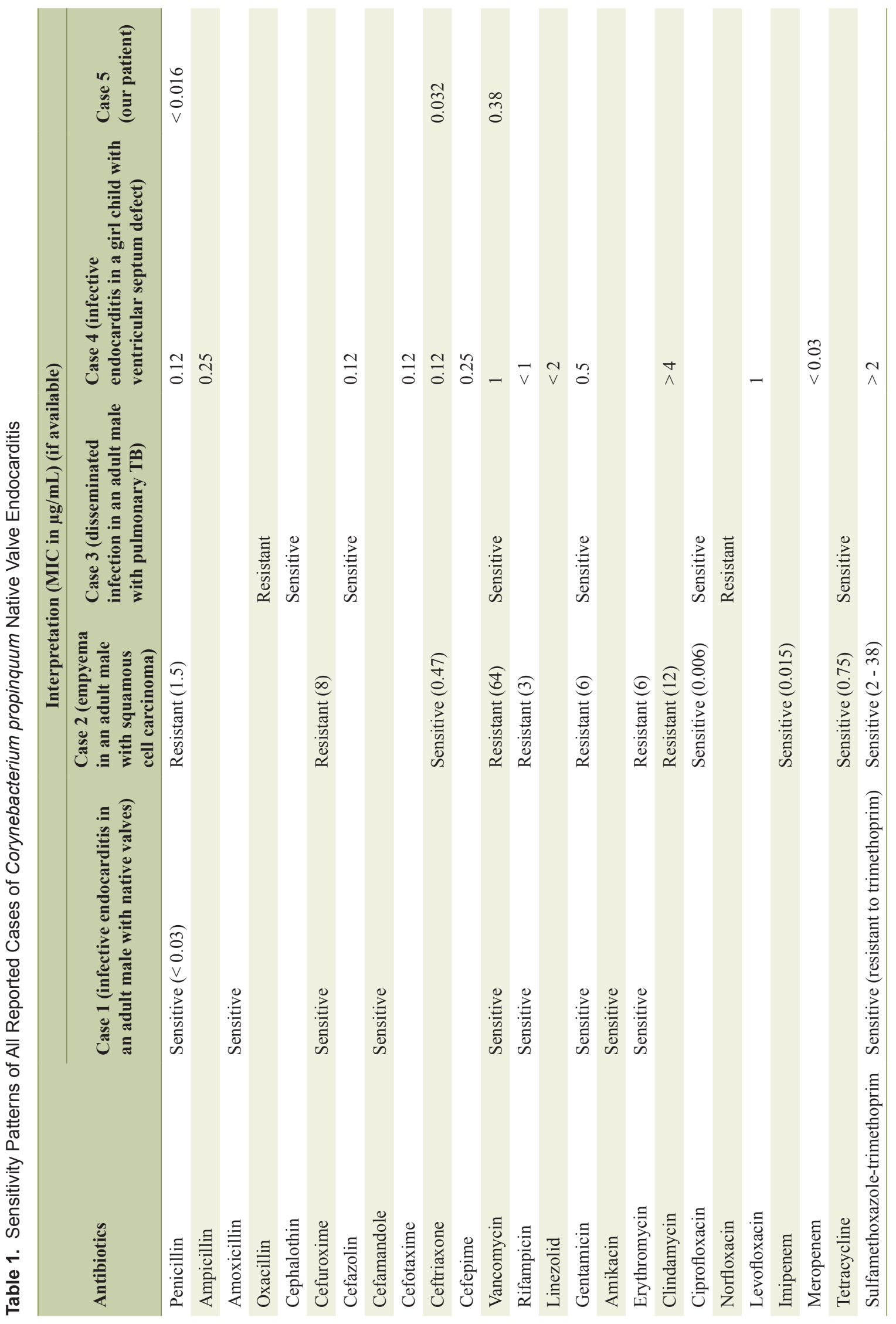

Articles (C) The authors | Journal compilation (C) J Med Cases and Elmer Press Inc ${ }^{\mathrm{TM}}$ | www.journalmc.org 
is rare, more so by non-diphtheria corynebacterium in native valves [1-4]. C. propinquum was previously called CDC group ANF-3. The current name was proposed by Riegel et al in 1993 [5]. C. pseudodiphtheriticum and CDC group ANF-1 are distinguished from the phylogenetically related $C$. propinquum by the fact that it is urease negative and reduces nitrates, respectively $[1,6]$. It is considered a saprophyte and has been isolated infrequently from skin, throat, and nasopharynx [5, 7]. An extensive search revealed only four prior reported cases of infection by this organism. Two of them were cases of infective endocarditis. One developed in the tricuspid valve of a 7-year-old girl with ventricular septum defect and ventriculoatrial communication [8], and the other in a 52-year-old man with a severely calcified, stenotic aortic valve [9]. To the best of our knowledge, this is only the second reported case of infective endocarditis in an adult caused by C. propinquum.

The other two cases included a disseminated infection with both bone marrow and blood cultures growing the organism in a 76-year-old man with pulmonary tuberculosis [10], and empyema in a 70-year-old man with squamous cell carcinoma [11].

There are no established guidelines regarding antibiotic therapy in these infections. It is thus recommended to report MIC results without interpretative criteria [6]. The resistance pattern in each of the reported cases varied and is summarized in Table 1.

In case 1 , the patient was initially treated with penicillin $\mathrm{G}$ for 6 weeks followed by valve replacement and cleaning out of an aortic root cavity with a thrombotic mass. He then received penicillin $\mathrm{G}$ for another 2 weeks and was successfully treated. In case 2 , a chest tube was placed and the patient was started on intravenous ceftriaxone and metronidazole. However, given his underlying malignancy, the focus of management was switched to palliative care. He expired shortly after. In case 3, the patient was successfully treated with 6 weeks of intravenous vancomycin in addition to anti-tuberculous regimen (isoniazid, rifampin, ethambutol, and pyrazinamide). Case 4 required a more complex approach. The patient was first treated with intravenous ceftriaxone for 3 days till sensitivity testing was completed. The patient was then treated with intravenous ampicillin and gentamicin for 4 and 2 weeks, respectively. A follow-up revealed persistently elevated C-reactive protein and persistent vegetations on echocardiogram. Due to concern for acquired penicillin resistance, she was treated again with 6 weeks of intravenous cephazolin and 3 weeks of intravenous gentamicin, with resultant resolution. In our case, we used 6 weeks of intravenous ceftriaxone as described above. This case highlights the lack of reliable treatment guidelines and the importance of continued reporting of infections by this rare organism as more cases of corynebacterial infective endocarditis are identified.

\section{Conflicts of Interest}

The authors declare no conflicts of interest.

\section{Grant Support}

None.

\section{References}

1. Funke G, von Graevenitz A, Clarridge JE, 3rd, Bernard KA. Clinical microbiology of coryneform bacteria. Clin Microbiol Rev. 1997;10(1):125-159.

2. Belmares J, Detterline S, Pak JB, Parada JP. Corynebacterium endocarditis species-specific risk factors and outcomes. BMC Infect Dis. 2007;7:4.

3. Bernard K. The genus corynebacterium and other medically relevant coryneform-like bacteria. J Clin Microbiol. 2012;50(10):3152-3158.

4. Schnell D, Beyler C, Lanternier F, Lucron H, Lebeaux D, Bille E, Bonnet D, et al. Nontoxinogenic corynebacterium diphtheriae as a rare cause of native endocarditis in childhood. Pediatr Infect Dis J. 2010;29(9):886-888.

5. Riegel P, De Briel D, Prevost G, Jehl F, Monteil H. Proposal of Corynebacterium propinquum $\mathrm{sp}$. for Corynebacterium group ANF-3 strains. FEMS Microbiol. 1993;113:229-234.

6. Martinez-Martinez L. Clinical significance of newly recognized coryneform bacteria. Rev Med Microbiol. 1998;9:55-68.

7. Coyle MB, Lipsky BA. Coryneform bacteria in infectious diseases: clinical and laboratory aspects. Clin Microbiol Rev. 1990;3(3):227-246.

8. Kawasaki Y, Matsubara K, Ishihara H, Nigami H, Iwata A, Kawaguchi K, Fukaya T, et al. Corynebacterium propinquum as the first cause of infective endocarditis in childhood. J Infect Chemother. 2014;20(5):317-319.

9. Petit PL, Bok JW, Thompson J, Buiting AG, Coyle MB. Native-valve endocarditis due to CDC coryneform group ANF-3: report of a case and review of corynebacterial endocarditis. Clin Infect Dis. 1994;19(5):897-901.

10. Dominquez D, Antony N. Disseminated Corynebacterium Propinquum (CDC group ANF 3) Infection in a Patient with Reactivated Tuberculosis. The Internet Journal of Infectious Diseases. 2008;7(1).

11. Babay HA. Pleural effusion due to Corynebacterium propinquum in a patient with squamous cell carcinoma. Ann Saudi Med. 2001;21(5-6):337-339. 